\title{
An Interoperable GIS solution for the public administration (extended abstract)
}

\author{
Artur Rocha, João Correia Lopes, Luís Bártolo, Rui Chilro \\ INESC-Porto, Rua Dr. Roberto Frias, No 378 4200-465 PORTO, Portugal, \\ \{ajrocha, jlopes, lbartolo, rchilro\}@inescporto.pt, \\ http://www.inescporto.pt
}

\begin{abstract}
Geographical information is of strategic importance, when comes to land use management decision-making and GIS are essential resources for the production of land use management instruments, commonly known as plans.

To build them, local authorities require multi-disciplinary teams with different competences and responsibilities. Many of these teams are external to the municipality and in the position of sub-contractors. With so many actors involved, the result is often a complex mesh of incoherent spatial data. Overcoming these problems often leads to huge overheads for the public administration.

This paper addresses a possible technical solution for this issue, based on international standards and profiting from the current state of technological development.
\end{abstract}

\section{Introduction}

Local authorities are responsible for innumerable decisions related to land use management. Some of these decisions must be taken in accordance with metropolitan/regional authorities and central government, and involve a set of complex tasks with a large volume of data of different nature and origin. Undoubtedly, geographical information (GI) is of strategic importance, when comes to land use management decision-making. Geographical information systems (GIS) are essential resources for the production of land use management instruments, commonly known as plans.

A report elaborated by the Instituto Nacional de Estatística (INE) refers that $63 \%$ of all plans executed by the local government are done by external companies, to whom some information must be transferred in a friendly way. Likewise, both external and internal access to this data must be granted according to specific profile definitions, suggesting the need for distributed access.

Project teams, often external and in the position of sub-contractors, encounter the same sort of problems during their intervention, despite of the plan they are working or the granularity of GI data. Most of these problems are related to the lack of a common spatial infrastructure and lead to serious accuracy 
and coherence issues. Overcoming these issues often leads to huge overheads for the public administration.

The delays in the development of a common Spatial Data Infrastructure (SDI) at the European level contribute to the chaos installed. Even if such infrastructure were to exist there would be a long way to go until it fits the regional or local level of detail.

This is the context in which municipalities, metropolitan and regional authorities have to keep producing their plans to support their daily land use decisions. As such, a spatially-enabled distributed work environment seems a natural solution to reduce the aforementioned overhead, creating the assets for a SDI at the local level.

This paper addresses a possible technical solution for this issue, based on international standards and profiting from the current state of technological development.

\section{Characterisation of the actual situation}

Local authorities land management decisions are based on heterogeneous data - from several sources, in different scales, projections and formats. Thus the decision-making process relies on data produced by several organizational units which frequently use different and incompatible systems, often resulting in poor integration and lack of data sharing.

Desirably, teams should be able to use their own data and be able to access data produced by others, in a constructive and cooperative way: in other words, a distributed shared environment where everyone can benefit from the combination of multiple information sources. The resulting environment is an information sharing network, where the most important feature is interoperability. Being the creation of data sources the most expensive part of any GIS effort, reusing spatial data seems a natural solution. However, there are difficulties both in sharing and in finding the appropriate spatial data[SDVC]:

- Institutional Impediments to Make Data Accessible: Assembling a multipurpose geographic data has additional costs (e.g. documentation, transfer, technical support, ...) that make it difficult to justify. So, the data produced today is mostly single-purposed and hard combine.

- Difficulty in the Discovery of Available Data Sources: even if the appropriate geographic data did exist, how would one find out about it?

- Difficulty in Assessing the Appropriateness of Data: Metadata is scarce or often inexistent and crucial to determine the appropriateness of data. The data producer, capture date, the time period it represents, the purpose why it was collected and a clear identification of the attributes are but a few examples of the records that must be kept.

- Technical problems in Data Transfer: For commercial reasons, software vendors have deliberately created obstacles to moving data from one proprietary application to another. Better communication protocols, storage media and 
data transfer standards have started to contribute to improve this situation, but for the present one needs to know a lot of general arcane computer lore to be able to scavenge through GIS data.

- Technical Problems in Conversion: Local authorities often use more than one GIS technology for specialized fieldwork, creating the need to convert data between systems. More often, the data available isn't in the desired format or needs to be used in a substantially different GIS data model (e.g. vector to raster). Some of these conversions involve the reinterpretation of the meaning of the data attributes; decisions and compromises must be made.

\section{Goal}

The strategic goal of this framework proposal is to improve access to geographical information resources for decision-makers as well as other involved parties. On a metadata point of view the system can be easily configured to support about any kind of information, but the use of standards will be enforced. The main characteristic of services described hereinafter is their truly distributed nature, as they may be located anywhere in the world and still be able to interoperate.

\section{Reference Architecture}

\subsection{Framework architecture}

The proposed architecture implements a typical 3-tier, basing its structure on the distributed model suggested by the OpenGIS and TC211 standards. This arrangement provides a high degree of flexibility in a distributed environment. By cleanly separating each layer, and using Geography Markup Language (GML) for the data transport, encoding and request formats, many individual participants can contribute to the network without the overhead of negotiating these agreements on a case-by-case basis. This is one of the advantages of the architecture. The global view of any data flow in this architecture comprehends a client requesting the map, data or service; a service that receives, processes and execute the request; and a tier that permits distributed access all over the system to transfer and integrate both ends. This tier may be an Object Request Broker (ORB) or based on Web Services.

A detailed view of each component fom this architecture follows.

\subsection{The Common tier}

For a correct data transfer, over a distributed and shared environment, there should be a common tier responsible for transportation of all data and request, even in heterogeneous environments.

This is by far the most important tier, since all the components need it's support. It is responsible to interconnect all services and, transfer requests and responses 


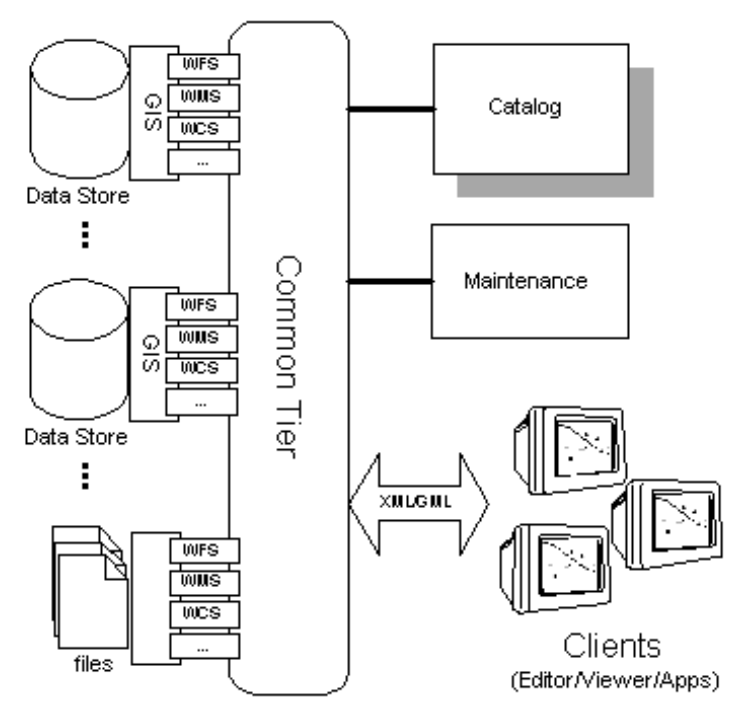

Figure 1. Framework architecture

between them. The data should be delivered making all the necessary adjustments between environments.

Additionally it must be able to connect to other nodes geographically distributed, using any arbitrary available Local Area Network (LAN), in a completely transparent way to the user. The only thing the user should know is that there is a service that shows all the available geographical data, and all other aspects of the architecture are transparent to him.

If there are any performance or security issues to satisfy, this layer should be able to compress and/or encrypt the data passed.

\subsection{The catalog}

The catalog service acts as the yellow pages of this system, a queriable list of all available data. After each service is started and initialised with the common tier, it must register itself in the catalog so that all its services be widely available. As suggested by the OpenGIS consortium, the catalog can derive is information from other catalogs, making it possible to create a hierarchy tree of catalogs. In this way, it is easier to manage all the available data and give access to data by, for example, access level, implement load balancing, fail-safe and/or redundant service and increase availability.

This service must implement methods for querying/searching geographical data based on type, area desired or word search. After choosing the desired data, the catalog should provide the location for the user to access them. 


\subsection{A simple client: the viewer}

The interactive viewer is one of the possible client services and is responsible for showing all the information selected by the user. It acts as a front-end to the system. The user, through the use of the viewer, accesses the catalog and chooses the data to see and, in a transparent step, the data is extracted transformed and represented.

The transparent process, quoted before, involves the use of the common tier to request and receive the data involved. This data is passed in GML ${ }^{1}$ and through the use of XSL Transformations (XSLT) is converted to Scalable Vector Graphics (SVG), the format chosen for visualization of Geographical data. The decision behind SVG was based on open source render formats without loss of detail and quality, flexibility and adaptability to almost any situation. With this front-end is possible to save the data on-to the desired device (remotely through one of services or in local disk), access geographical information by selecting any feature on the map, add red-layers and in future geographical objects.

\subsection{The data providers}

The data is accessed through any of the services described next. These services provide data on a standard way (OpenGIS) allowing flexibility and ease for future developments and integration with other systems.

Any developed component may implement more than one service or even all, providing that all the services are registered individually in the catalog.

These services implement OpenGIS standards so, a simple front-end, with oneto-one method wrap over all services, may provide the information available on each of the wrapped service, in OpenGIS standard to the web (i.e. a servlet).

The WMS service. The simplest service of all, is described in the specification of OpenGIS and TC211 under the name of Web Map Server (WMS), and is intended to deliver an image map, customized by the client request, in this particular case, via the common layer.

The client can decide on the output image format requested from a list of formats defined in the standard (image/gif, image/jpg, image/tiff, image/geotiff, image/png, image/ppm and image/wbmp).

The metadata with the map and services available can be queried at any time and the subsequent response is sent on XML format.

The WFS service. Web Feature Servers are designed to provide access to (and interaction with) non-image data collections.

OGC has followed one of the principle axioms of Extensible Markup Language (XML) in its Web services architecture - the separation of structure, style and content. Web Feature Services, specified by this consortium, enable sophisticated, Web-based geoprocessing. Yet it doesn't necessarily mean the user must

\footnotetext{
${ }^{1}$ The possible use of GML 3.0, approved in 06-Feb-2003 by OpenGIS is in review.
} 
deal with anything more complex than the interface in basic WMS applications. Generally, WFS operations support INSERT, UPDATE, DELETE, QUERY and DISCOVERY of geographic features and the solicited data is passed in standard text/xml format.

The WCS service. This is a very recent service ${ }^{2}$ and is intended to simple coverages (defined on some regular, rectangular or tessellation grid of space) and anticipates other coverage types defined in the OpenGIS Abstract Specification. The specification document explains how "WCS serves to describe, request, and deliver multi-dimensional coverage data over the World Wide Web"[WCS02]. This service is based on this specification and receive requests and delivers coverage data. Web Coverage Service provides access to intact (unrendered - as needed for client-side rendering) geospatial information, multi-valued coverages, and input into scientific models for advanced rendering and visualization clients.

\subsection{Maintenance.}

Anyone responsible for the management of all system needs some tools to maintain and audit the system. This component tries to respond to this need. It is able to start and stop any service or interrupt any damaged or malicious access. In future developments, this component may be enhanced to take statistics of the system and help on pro-active maintenance, load balancing, service replication, security, profiling, etc.

\section{Conclusions and Future Work}

This paper aims to conclude on the adequability of the proposed architecture to help solving the issues initially proposed.

A prototype driven approach is being used to assess the framework and identify problems on an early development stage, resulting in a evolving architecture that starts to prove its strengths - interoperable and distributed access to several GI collections (for viewing), through a catalog.

So far, the preliminary tests have served to provide a proof of concept for the implemented services, even if on an embrionary development stage.

At this point we are led to believe that this framework implementation will contribute to resolve the issues initially proposed, seeming that there is almost no practical limit to scaling or extending this framework architecture to take advantage of additional data resources, services or applications.

\section{References}

[SDVC] site: http://www.sdvc.uwyo.edu/metadata/cote.html

\footnotetext{
${ }^{2}$ RFC for technologies and needed interfaces launched in 24-Dec-2002.
} 
[WCS02] Open Gis Consortium, Request for Comment - OpenGIS Web Coverage Service, http://www.opengis.org/techno/02-024r1.pdf, (December 2002)

[OGCSpec02] Open Gis Consortium, OpenGIS Abstract Specification, http://www.opengis.org/techno/abstract.htm, (September 2002)

[OGCCAT02] Open Gis Consortium, OpenGIS Catalog Services Implementation Specification v1.1.1,

http://www.opengis.org/techno/specs/02-087r3.pdf

[OGCWMS01] Open Gis Consortium, OpenGIS Web Map Service Interfaces Implementation Specification v1.1.1, http://www.opengis.org/techno/specs/01-068r3.pdf

[OGCWFS02] Open Gis Consortium, OpenGIS Web Feature Service Implementation Specification v1.0, http://www.opengis.org/techno/specs/02-058.pdf

[OGCGML02] Open Gis Consortium, OpenGIS Geography Markup Language (GML) Implementation Specification c3.0, http://www.opengis.org/techno/documents/02023r4.pdf

[OMGCORBA] Object Management Group, Catalog of OMG Specifications, http://www.omg.org/technology/documents/spec_catalog.htm

[NEWCOMER02] Newcomer, Eric: Understanding Web Services, Addison-Wesley, 2002 ISBN: 0-201-75081-3 